\title{
The true colors of autophagy in doxorubicin-induced cardiotoxicity (Review)
}

\author{
BIN XIAO ${ }^{1,2 *}$, LANG HONG ${ }^{1,3^{*}}$, XINYONG CAI $^{1,3^{*}}$, \\ SONGBO MEI ${ }^{1,2}$, PING ZHANG $^{4}$ and LIANG SHAO ${ }^{1,3}$ \\ ${ }^{1}$ Department of Cardiology, Jiangxi Provincial People's Hospital; ${ }^{2}$ Medical Graduate School of \\ Nanchang University; ${ }^{3}$ Jiang Xi Provincial Institute of Cardiovascular Diseases; ${ }^{4}$ Department of \\ Neurology, Jiangxi Provincial People's Hospital, Nanchang, Jiangxi 330006, P.R. China
}

Received August 17, 2018; Accepted June 13, 2019

DOI: $10.3892 / 01.2019 .10576$

\begin{abstract}
Patients with cancer receiving doxorubicin-based chemotherapy often have to stop taking the drug due to its cardiotoxicity and therefore lose out on the beneficial effects of its potent antitumor activity. Doxorubicin has been demonstrated to damage cardiomyocytes via various mechanisms, including accumulation of reactive oxygen species (ROS), DNA damage and autophagy dysfunction. The present review focuses on autophagy, describing the general process of autophagy and the controversy surrounding its role in doxorubicin-induced cardiotoxicity. In addition, the associations between autophagy and apoptosis, ROS, DNA damage and inflammatory processes are discussed. In the future, it will be useful to further elucidate the process of autophagy and reveal its association with various pathological processes to develop effective strategies of preventing doxorubicin-induced cardiotoxicity.
\end{abstract}

\section{Contents}

1. Introduction

2. Interactions between autophagy and apoptosis

3. Complex role of autophagy in doxorubicin-induced cardiotoxicity

4. Oxidative stress and autophagy in doxorubicin-induced cardiotoxicity

5. DNA damage and autophagy in doxorubicin-induced cardiotoxicity

Correspondence to: Dr Liang Shao, Department of Cardiology, Jiangxi Provincial People's Hospital, 92 Aiguo Road, Donghu, Nanchang, Jiangxi 330006, P.R. China

E-mail: shaoliang021224@hotmail.com

*Contributed equally

Key words: doxorubicin, cardiotoxicity, autophagy
6. Inflammation and autophagy in doxorubicin-induced cardiotoxicity

7. Conclusions

\section{Introduction}

Anthracyclines are a group of highly effective chemotherapeutic agents used in the treatment and management of various cancer types (1). The anthracycline doxorubicin (DOX) is a potent and broad-spectrum antineoplastic agent used to treat patients with leukemia, lymphoma, sarcoma or breast cancer (2). However, cardiovascular complications are well-documented side-effects of anthracyclines. DOX can impair the pumping function of the left ventricle and lead to dilated cardiomyopathy and congestive heart failure (HF), resulting in the termination of the treatment and worsening the prognosis of the patient $(2,3)$. Furthermore, the risk of developing subsequent cardiotoxicity increases with the dose of DOX received. A previous study demonstrated that patients who received a cumulative dose of $400 \mathrm{mg} / \mathrm{m}^{2}$ DOX exhibted a $5 \%$ increase in the risk of HF. This risk was further increased by 26 and $48 \%$ when doses of 550 and $700 \mathrm{mg} / \mathrm{m}^{2}$ DOX were administered, respectively (4). From a clinical pathology point of view, DOX-treated patients can exhibit extensive cardiac remodeling, including vast cytoplasmic vacuolization, sarcoplasmic reticulum swelling and myofibrillar disarray (5). To the best of our knowledge, currently no drug has been confirmed to effectively prevent DOX-induced cardiotoxicity without diminishing its antitumor activity, which is predominantly due to a lack of understanding regarding the mechanisms underlying DOX-induced pathology.

The broad mechanisms underlying DOX-induced cardiotoxicity are the following: i) oxidative stress, ii) DNA damage and its subsequent effects, and iii) autophagy dysfunction or impaired autophagic flux (1). Autophagy, which has gradually been recognized as a physiological process essential for maintaining cellular homeostasis, is not limited to a single signaling pathway $(1,4)$. It is a dynamic, multistep biological process that can be regulated by numerous factors at a number of stages and overlaps with other cellular processes (6). The regulatory pathways of autophagy remain to be fully elucidated 
and a number of studies have reported conflicting evidence regarding the role of DOX in the regulation of autophagy in cardiac cells $(7,8)$. Therefore, it is essential to investigate these regulatory pathways, as well as other mechanisms associated with autophagy, to improve understanding of the role of autophagy in DOX-induced cardiotoxicity and assist with the development of drugs to prevent this pathological condition.

\section{Interactions between autophagy and apoptosis}

Programmed cell death (PCD) refers to a process in which cells follow a defined intracellular program and commit suicide in response to physiological or pathological conditions (9). Based on the death mechanisms, PCD can be divided into caspase-independent and caspase-dependent cell death, the former including autophagic programmed cell death (9). The present section focuses on autophagy, which is the death of numerous organelles, apoptosis, which is the death of the whole cell, and their association.

Autophagy is a catabolic process involving the degradation of cellular components $(1,10)$. Autophagy is controlled by autophagy-related genes (ATGs) and lysosomal proteolytic mechanisms, which are activated in response to different types of metabolic stress, including hypoxia, reactive oxygen species (ROS) accumulation and nutritional or energetic deficiency $(11,12)$. The five core steps of autophagy include initiation, nucleation, expansion, maturation, and degradation (13). Each of these steps have corresponding regulators, including serine-threonine kinase Unc-51-line kinase-1 (ULK1), ATG13 and focal adhesion kinase family interacting protein of $200 \mathrm{kD}$ (FIP200) complexes, which are important for the promotion of autophagy (Fig. 1). Adenosine monophosphate-activated protein kinase (AMPK) and mammalian target of rapamycin (mTOR) signaling pathways constitute the regulatory core of autophagy; however, it is also regulated by a number of other signaling pathways and mediators, including Beclin-1, B-cell lymphoma 1 (Bcl-2), phosphoinositide-3-kinase (PI3K) and p53 proteins $(14,15)$. Consistently, numerous studies have demonstrated that the AMPK and mTOR signaling pathways promote and inhibit autophagy, respectively $(1,16)$. mTOR is regulated by major upstream signals in the PI3K-Akt-mTOR pathway. In more detail, intracellular or extracellular stress can activate the PI3K receptor, which catalyzes the conversion of phosphatidylinositol 4,5-biphosphate into phosphatidylinositol 3,4,5,-triphosphate (PIP3), which is a catalytic reaction that can be reversed by phosphatase and tensin homolog. In co-operation with phosphoinositide-dependent kinase-1, PIP3 then further activates Akt. In turn, activated Akt increases the activity of Ras homolog enriched in brain, which upregulates mTOR activity, and inhibits autophagy initiation by promoting ULK1 phosphorylation (17,18) (Fig. 2).

Conversely, a number of studies have demonstrated that when the body lacks nutrients or energy, an increased adenosine monophosphate/ATP ratio can activate AMPK, thereby inhibiting mTOR, weakening the inhibition of ULK1 and finally initiating autophagy $(15,16)$. As a sensor in autophagy regulation, mTOR receives information from different upstream signal molecules and accordingly regulates downstream signal molecules, including p70S6K, eukaryotic translation initiation factor 4E-binding protein 1 , ULK1, lipin-1 and peroxisome proliferator-activated receptor $\gamma$ coactivator $1 \alpha$, which produce their own biological effects (19). The absolute increases and decreases in mTOR activity are therefore determined by the ratio of various factors or stimuli. In other words, autophagy regulation may involve co-occurring activation and inhibition of mTOR, depending on which of the respective regulatory factors predominate. Autophagy regulation is indeed a complicated process. While a basal level of autophagy is necessary to maintain cellular homeostasis, autophagic cell death without nuclear pyknosis is initiated when autophagy gets overactivated and exceeds a certain threshold $(10,20)$.

Apoptosis similarly serves a fundamental role in maintaining cellular homeostasis and is also regulated by Bcl-2 and p53 genes, which are closely associated with autophagy $(19,21)$. Autophagy and apoptosis respond to similar types of stress and signals, including a high intracellular $\mathrm{Ca}^{2+}$ concentration, ROS accumulation, toxins, growth factors and hormonal stimulation (22). Furthermore, the same proteins can promote autophagy and apoptosis mechanisms, in their respective processes. For example, under cellular stress conditions, autophagy-related 5 (ATG5), a protein necessary for the formation of the autophagy precursor, is cleaved by a cysteine protease and serves a key role in the initiation of apoptosis. Notably, under autophagy-inducing conditions, Beclin-1, which normally binds to Bcl-2 and inhibits autophagy, is competitively displaced and subsequently promotes the autophagic process (21). Altogether, the promotion and inhibition of autophagy and apoptosis contribute to the difference between cell death and survival. In the case of an increased proportion of apoptosis, caspases, the primary mediators of apoptosis, can cleave numerous key autophagy proteins, for example, caspase- 3 and -8 can cleave PI3K and ATG3, respectively (21). Strengthening this process will inevitably lead to cell destruction. On the other hand, resveratrol can induce autophagy via the kinase triad, AMPK, mTOR and ULK1, resulting in a decreased proportion of apoptosis (23-25).

Given that the regulatory pathways of autophagy are complex and intertwined with those of apoptosis, it remains challenging to elucidate the precise role of autophagy under drug treatments conditions, including DOX.

\section{Complex role of autophagy in doxorubicin-induced cardiotoxicity}

The autophagic process involves the formation of a structure sealed by a lipid bilayer membrane called an autophagosome (26). This autophagosome can fuse with a lysosome to form an autolysosome, which can degrade damaged and redundant organelles or invading microorganisms $(1,10)$. To date, to the best of our knowledge, 38 ATGs have been identified to regulate different stages of autophagy in yeast. In mammals, part of this network is conserved, but a number of additional genes are involved in the regulation of autophagy (27).

The function of ATG products, particularly their capacity to interact with other autophagy regulators at different stages of the process, is predominantly achieved by proteolysis and protein post-translational modifications, including phosphorylation, glycosylation, ubiquitination, methylation, acetylation and lipidation (27). For example, in mammals, ATG13 and FIP200 are involved in the formation of a complex required 


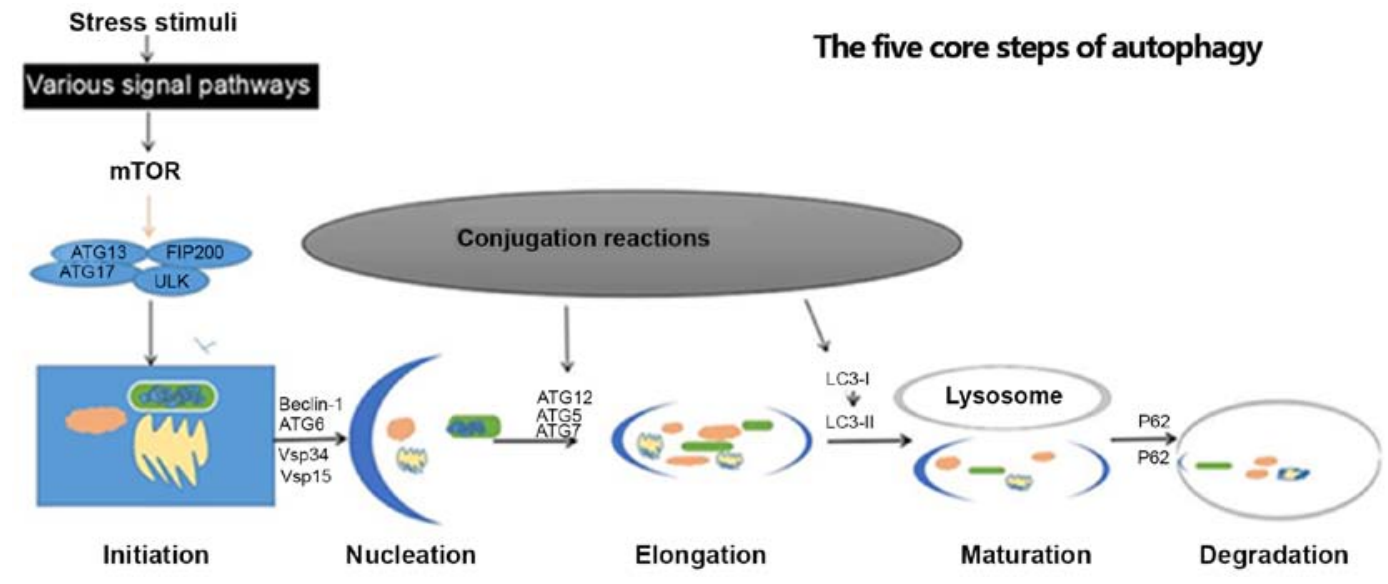

Figure 1. Five core steps of autophagy: Initiation, nucleation, elongation, maturation and degradation. This process is induced by various stress stimuli, including inhibition of mTOR. In initiation, the organelles to be degraded are gradually wrapped. Subsequently, an autophagosome is formed and cytosolic components are sequestered and characterized by a LC3-II-positive double membrane structure. In the final step, cytosolic components are degraded in an autolysosome. ATG, autophagy related gene; LC3, light chain 3; mTOR, mammalian target of rapamycin; ULK1, serine-threonine kinase Unc-51-line kinase-1; Vsp, vacuolar protein sorting; FIP200, focal adhesion kinase family interacting protein of $200 \mathrm{kD}$.

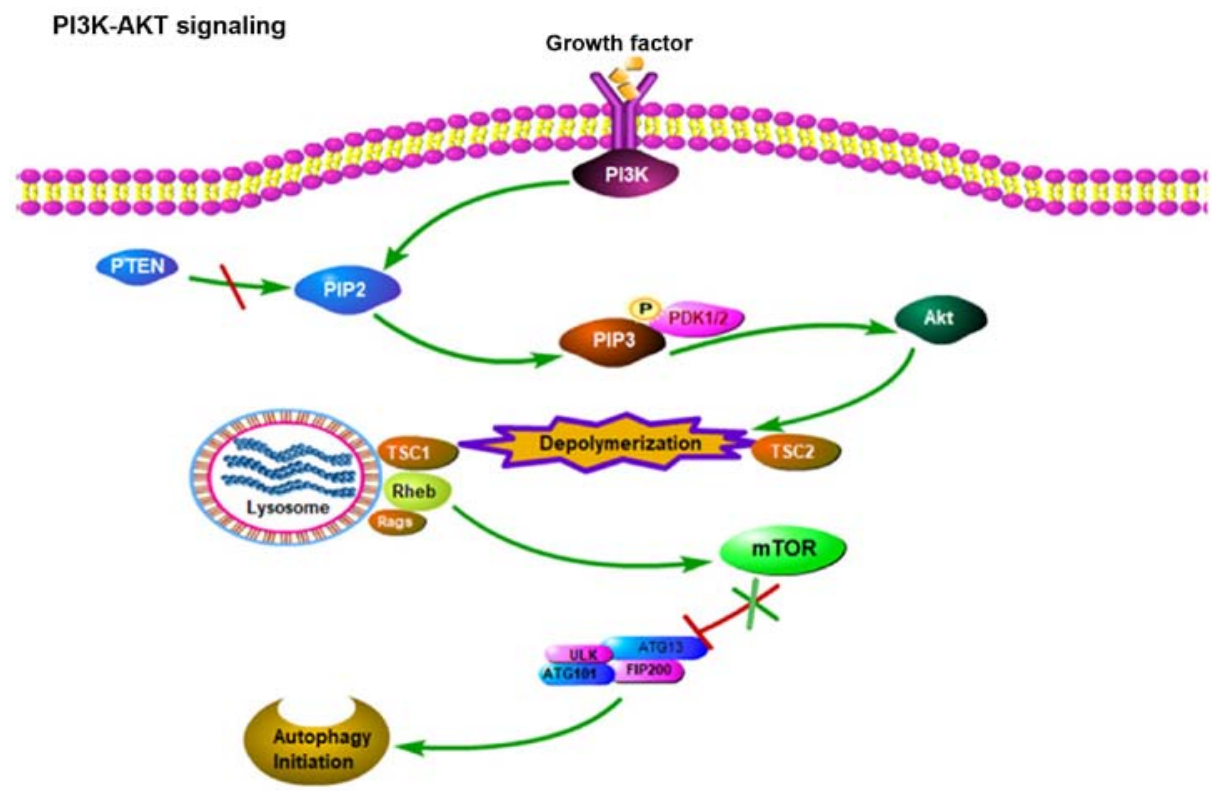

Figure 2. PI3K-AKT signal path diagram: Intracellular and extracellular factors activate the receptor of PI3K, which in turn catalyzes the conversion of PIP2 to PIP3, a catalytic reaction that is reversed by PTEN. PIP3 further activates Akt with the cooperation of PDK1/2. Activated Akt increases the activity of Rheb, which in turn upregulates mTOR activity, which inhibits autophagy initiation by promoting ULK1 phosphorylation. PI3K, phosphoinositide-3-kinase; PIP2, phosphatidylinositol 4,5-biphosphate; PIP3, phosphatidylinositol 3,4,5,-triphosphate; PTEN, phosphatase and tensin homolog; PDK1, phosphoinositide-dependent kinase 1 and 2; TSC, tuberous sclerosis 1; Rheb, Ras homolog enriched in brain; Rags, recombination-activating genes; mTOR, mammalian target of rapamycin; ATG, autophagy related gene; ULK, serine-threonine kinase Unc-51-line kinase; FIP200, focal adhesion kinase family interacting protein of $200 \mathrm{kD} ;$ P, phosphorylated.

for the initiation of autophagy. By recruiting the Beclin-1 and vacuolar protein sorting 34/15 proteins, ATG13 is involved in the formation and regulation of a class III PI3K complex essential for the nucleation step (28). During the expansion step, ATG3, ATG4, ATG7, ATG10 and ATG16 serve important roles in promoting the formation of two essential ubiquitin-like (Ubl) conjugation systems, ATG12-ATG5 and light chain 3 (LC3)-phosphatidylethanolamine (27,29). These two systems contribute to the amplification of phagocytic cells. In addition to the Ubl conjugation systems, the ATG9-mediated cycling systems have been demonstrated to contribute to the elongation of the phagophore and numerous other ATG products also function in the late steps of autophagy (27). In the later steps of maturation and degradation, the autophagosomes move to and fuse with lysosomes in a kinesin-dependent manner (13). Subsequently, the cargo is degraded into macromolecules and released into the cytoplasm to be reused. A number of cytokines are also involved in the regulation of this process $(10,16)$. However, the role of ATG genes in this context remains to be fully investigated.

DOX treatment has adverse effects on multiple tissues and organs of the body, and primarily affects the heart due to induced DNA damage, swelling of the sarcoplasmic reticulum, cytoplasmic vacuolization, intracellular calcium imbalance 


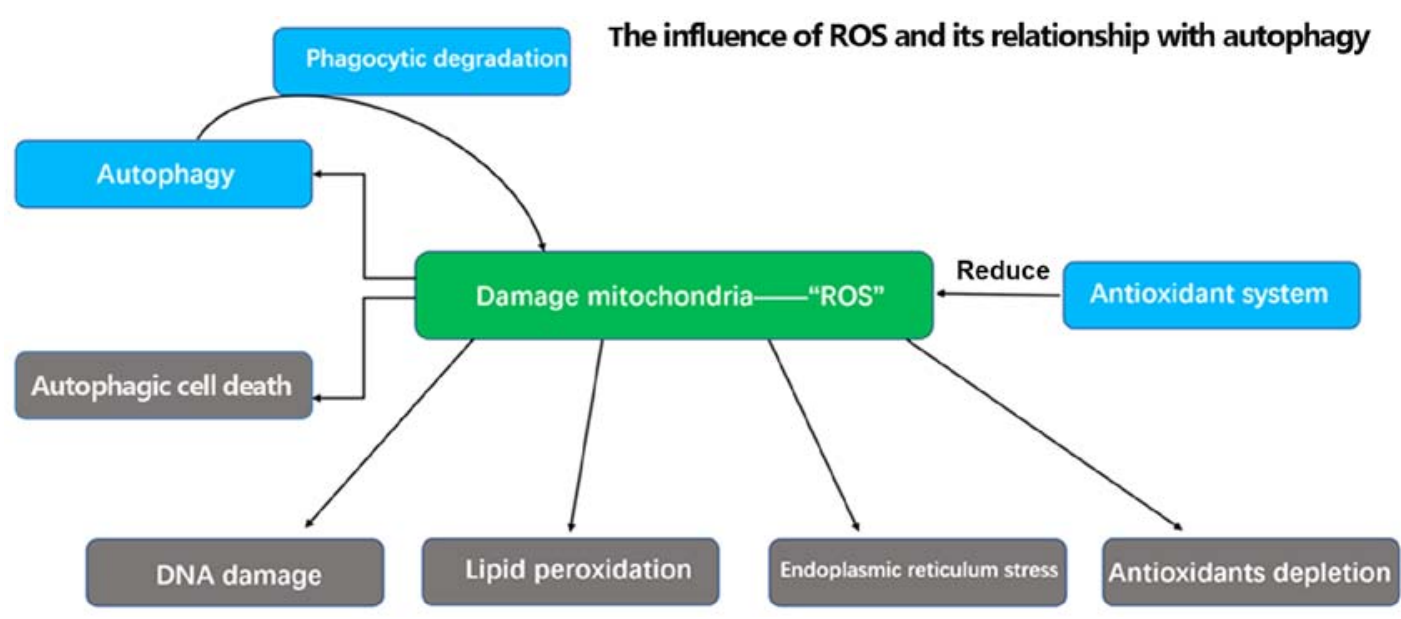

Figure 3. Influence of ROS and its association with autophagy. Damaged mitochondria represented by the green box produce excessive ROS that cause DNA damage, lipid peroxidation, endoplasmic reticulum stress, antioxidant depletion (shown in the gray box) and autophagy (shown in the blue box). This in turn promotes the phagocytosis of damaged mitochondria, reducing ROS levels from the source, indicated by the curved arrow. However, overwhelming levels of ROS can also trigger autophagic cell death (shown in the gray box). ROS, reactive oxygen species.

and myofibrillar disarray, which are observed in microscopic observations of the pathological structures (5). In addition to DOX itself, the pathological changes also cause autophagy dysfunction via the inhibition and activation of the AMPK and p38-mitogen-activated protein kinase (MAPK) pathways, respectively (30). Notably, numerous studies $(7,8,25,31)$ have presented conflicting conclusions, which suggests that autophagy may serve a dual role in DOX-induced cardiotoxicity.

Light chain 3 (LC3), a central protein in the autophagy pathway, contributes to substrate selection and autophagosome biogenesis. By inhibiting the Beclin-2 protein,DOX canincrease the protein expression level of LC3, which leads to cardiomyocyte death (32). Furthermore, in vitro assays have demonstrated that DOX can upregulate the pro-autophagy protein Beclin-1 and induce cardiomyocyte apoptosis (7). This indicates that the upregulation of the level of autophagy serves an adverse role in DOX-induced cardiotoxicity. Inconsistently, it has also been demonstrated that strengthening the level of autophagy via the AMPK/mTOR/ULK1 signaling pathway can improve the survival of cardiomyocytes (25). Furthermore, aspalathin can attenuate DOX-induced cardiotoxicity by activating the AMPK pathway, which increases the level of autophagy, while reducing the extent of $\mathrm{p} 53 / \mathrm{mTOR} / \mathrm{p} 62$ signaling (8). In summary, these data suggest an opposite conclusion as aforementioned, which is that upregulating autophagy can attenuate DOX-induced cardiotoxicity. Furthermore, DOX has also been demonstrated to inhibit autophagy by activating the E2F transcription factor 1 (E2F1)/mTOR complex and further induces apoptosis by activating the E2F1/AMPK 22 pathway (31). The conflicting results of these studies are most likely due to the observation of different, intertwined pathways in each set of experiments. It is plausible that the differences in the conclusions of these studies may come from differences in the experimental reagents, animal models and other experimental conditions, including the dose of DOX used. Therefore, it is conceivable that DOX inhibits autophagy in a mouse model, whereas, under different conditions, an increase of autophagy is observed in a rat model.
Therefore, the present study proposes that the apparent complexity of the role of autophagy in DOX-induced cardiotoxicity is due to the existing associations between autophagy and other physiological and pathological processes (33).

\section{Oxidative stress and autophagy in doxorubicin-induced cardiotoxicity}

The large accumulation of ROS caused by DOX, a process that predominantly occurs in the mitochondria and cytoplasm, has long been recognized as the main source of cardiac damage. Under DOX treatment conditions, hydrogen peroxide and superoxide radicals are formed following the induction of endogenous oxidases. This process ultimately leads to the production of the highly reactive and toxic hydroxyl radical by the iron-catalyzed Haber-Weiss reaction $(1,34)$. These radicals can react with proteins, lipids, DNA molecules and endogenous anti-oxidants, which causes protein oxidation, lipid peroxidation, DNA damage and antioxidant depletion, respectively (33). Furthermore, ROS can result in the activation of the AMPK/mTOR signaling pathway or ULK1 kinase, which increases autophagy (35). Indeed, previous studies have reported that treating cells with the ROS scavenger $\mathrm{N}$-acetylcysteine can reduce ROS production and autophagy $(36,37)$. Notably, it is well documented that ROS, like other external stimuli, including hypoxia, starvation and tumor necrosis factor- $\alpha$ (TNF- $\alpha$ ), can affect the autophagy processes (38). For example, ATG4 is an autophagic protease with an active cysteine residue regulated by intracellular redox reactions. In a reducing environment, ATG4 can serve its unique role in promoting the formation of autophagosomes. However, in DOX-induced cardiotoxicity conditions, ATG4 protease activity is inhibited by the oxidization of cysteine residues (39). These observations illustrate the complex and close association between autophagy and ROS.

It is worth noting that overwhelming levels of ROS can trigger autophagic cell death (Fig. 3). Furthermore, previous studies have demonstrated that redox signaling is not the only mechanism that links autophagy and ROS. When 


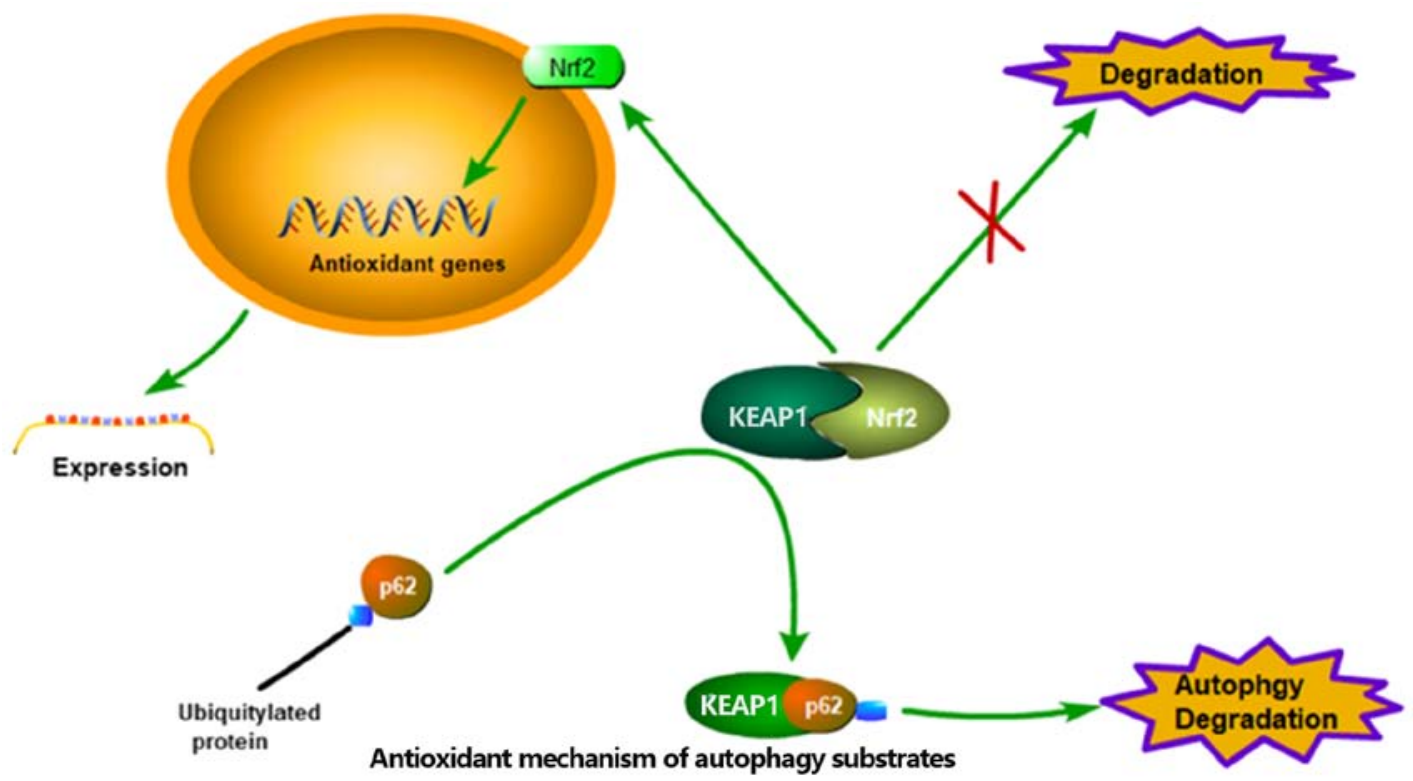

Figure 4. Antioxidant mechanism of autophagy substrates. p62, which bound to ubiquitylated protein aggregates, can undergo phosphorylation and leases KEAP1 from Nrf2 and Nrf2 transfers to the nucleus as a transcription factor instead of being degraded. Ultimately, Nrf2 transactivates the transcription and expression of antioxidant genes in the nucleus, and p62 is degraded by the autophagosome with KEAP1. KEAP1, Kelch-like ECH-associated protein 1; Nrf2, nuclear factor-like 2 .

bound to ubiquitylated protein aggregates, p62, an autophagy substrate used as a reporter of the degradation process in autophagosomes, can undergo phosphorylation on ser351 in a redox-independent manner. Phosphorylated p62 then serves an important regulatory role in the dissociation of the Kelch-like ECH-associated protein 1-nuclear factor-like 2 (Nrf2) complex, allowing Nrf2 to translocate to the nucleus and act as a transcription factor instead of being degraded. Subsequently, nuclear Nrf2 can transactivate anti-oxidation genes, driving their expression and ultimately attenuating the intracellular ROS levels (Fig. 4) (40-42).

\section{DNA damage and autophagy in doxorubicin-induced cardiotoxicity}

With a number of studies (43-48) being published in the domain of onco-cardiology, the associations between DOX and DNA damage are becoming increasingly apparent. Previous studies have suggested that DOX, in a topoisomerase II $\beta$-dependent manner, induces the formation of DNA double-strand breaks (DSBs), which can lead to ROS formation, mitochondriopathy and apoptosis of cardiomyocytes $(43,44)$. However, other studies investigating the molecular basis of anthracycline-induced cardiotoxicity, using knockout mice, have ruled out a direct involvement of topoisomerase II $\beta$ (43-46). Additionally, it has been suggested that DNA damage can initiate autophagy through multiple pathways. In the context of DOX-induced DNA damage, the activity of the $\mathrm{p} 53$ tumor suppressor gene is enhanced, and the sestrin 1 and sestrin 2 proteins can subsequently activate AMPK, thereby contributing to the initiation step of autophagy (47). Furthermore, in a different pathway, the p53 protein can also activate liver kinase B1 and thus induce autophagy via AMPK (48).

Notably, autophagy has a significant impact on the DNA damage response, by affecting the homologous recom- bination (HR) and non-homologous end joining (NHEJ) pathways, which are the two main DSB repair pathways (49). Previous studies have demonstrated that autophagy-deficient cells exhibit markedly diminished phosphorylation levels of checkpoint kinase 1, a critical mediator kinase in the HR pathway $(50,51)$. Consequently, these autophagy-deficient cells become highly dependent on the NHEJ pathway to perform DSB repair. However, in the long run, this continued dependence on NHEJ results in loss of nucleotides and gross chromosomal rearrangements, including translocations, and eventually causes a detrimental accumulation of DNA damage leading to cell death. As early as 2007, a study had established an association between autophagy and DNA damage. When the expression of BECN1, the gene coding for the autophagy protein Beclin 1 in mammals, is downregulated or completely abrogated, the autophagic survival response to DNA damage is severely impaired, which allows the cells to die by apoptosis (52). The combined data of numerous studies have led to the conclusion that stress-induced autophagy limits the amount of DNA damage and thus contributes to maintaining genome integrity. In the future, continuous investigations of the associations between autophagy and DNA damage will assist with the development of new therapeutic strategies.

\section{Inflammation and autophagy in doxorubicin-induced cardiotoxicity}

Previous studies have reported that acute and chronic myocarditis, inflammatory diseases, occur in a dose-dependent manner in patients receiving DOX treatment (4). Inflammation is a factor reported to be involved in DOX-induced cardiotoxicity and results from the combined action of numerous regulatory mechanisms (53). Indeed, in patients with cancer who exhibit DOX-associated adverse effects, a large number 
of cytokines, including interleukin-1 and TNF- $\alpha$, are released, which triggers various signaling pathways, including the nuclear factor- $\mathrm{\kappa B}(\mathrm{NF}-\kappa \mathrm{B})$ and $\mathrm{p} 38-\mathrm{MAPK}$ pathways, and the subsequent inflammatory cascades $(54,55)$.

These inflammatory signaling pathways are understood to have close associations with the autophagy pathway. NF- $\mathrm{KB}$ acts as a key regulator of the inflammatory response and is kept in the cytoplasm in an inactive state by the inhibitor of $\kappa \mathrm{B}$ protein. IKB kinase (IKK) interacts with TGF- $\beta$-activated kinase 1 and its cofactors TAK1 and MAP3K7-binding protein 2 and 3 to form a complex that degrades IкB $\alpha$. Activated NF- $\kappa \mathrm{B}$ can then translocate to the nucleus and induce an inflammatory response $(53,54,56)$. Notably, the TAK1 cofactors transforming growth factor- $\beta$-activated kinase 1-binding protein $2 / 3$ can also bind to Beclin-1 and induce autophagy (57). Therefore, the IKK-NF- $\mathrm{BB}$ pathway and part of the autophagy pathway are in an interdependent equilibrium association. Furthermore, under autophagy-deficient conditions, p62 overexpression activates IKK in human keratinocytes in a toll-like receptor-dependent manner, thereby activating $\mathrm{NF}-\mathrm{\kappa B}$ and triggering an inflammatory response. Conversely, p62 is degraded to inhibit the NF- $\mathrm{KB}$ signaling pathway when autophagy is upregulated (58).

In the context of DOX chemotherapy, high levels of NF- $\kappa \mathrm{B}-\mathrm{p} 65$ phosphorylation contribute to the nitric oxide-induced phosphorylation/activation of AMPK. In turn, the AMPK/mTOR signaling pathway can enhance autophagy, which inhibits the NF- $\mathrm{KB}$ signaling pathway and downregulates subsequent inflammatory cytokine responses (54). Therefore, upregulation of autophagy can serve an anti-inflammatory role. Of note, similar effects have been observed in a study associated with the treatment of rheumatoid arthritis (59).

In summary, these findings clearly demonstrate that autophagy serves an anti-inflammatory role and can exhibit additional beneficial effects in the context of doxorubicin-induced cardiotoxicity.

\section{Conclusions}

Doxorubicin causes cardiotoxicity through multiple mechanisms, including DNA damage, ROS accumulation, lipid peroxidation, energy deficiency, mitochondrial destruction, lysosomal destruction, calcium disorder and autophagy dysfunction $(1,4)$. These mechanisms are not entirely independent, as they occur simultaneously and interact with each other $(1,4)$. The exact role of autophagy is not fully understood, due to a large number of associated signaling pathways, the association between regulatory factors and the overlap with other mechanisms $(10,16)$. Apart from the effects on autophagy of ROS, DNA damage and inflammation, total calcium signaling can also affect the proximal and distal steps of the autophagic flux (60).

Furthermore, it is difficult for a single reporter to reflect the status of autophagy accurately and the majority of studies do not adequately consider the influence of external factors in their experimental setup. This has led to contradicting conclusions regarding the role of autophagy in doxorubicin-induced cardiotoxicity. Instead of monitoring a single indicator, the use of multiple methods to assess the status of autophagy would further our understanding of its entire regulatory process and assist with understanding how DOX induces changes in the regulation of autophagy.

Although the role of autophagy in DOX-induced cardiotoxicity remains controversial, numerous studies have reported that autophagy appears to be beneficial, by reducing the level of ROS, promoting the repair of damaged DNA, counteracting the effects of inflammation effects and protecting cardiomyocytes from apoptosis $(23,40,41,49,58)$.

As the number of studies continues to increase, there are numerous multi-faceted issues to resolve, including: i) the presence of other types of autophagy, including chaperone-mediated autophagy, which exhibit an unknown effect on cardiomyocytes; ii) the associations between doses or concentrations of DOX and the level of autophagy; and iii) how to modulate autophagy to attenuate cardiotoxicity without triggering autophagic cell death. Addressing these questions is not only essential to assist with the selection of drugs to antagonize the cardiotoxicity induced by chemotherapeutic drugs but also to avoid resistance to chemotherapeutic drugs and ultimately prevent and treat cancer.

\section{Acknowledgements}

Not applicable.

\section{Funding}

This study was funded by the National Natural Science Foundation of China (grant no. 81300115)

\section{Availability of data and materials}

Not applicable.

\section{Authors' contributions}

BX, LH and XC drafted the article and revised the manuscript. $\mathrm{SM}$ and $\mathrm{PZ}$ participated in the manuscript's revision and gave some suggestions for important content. LS proposed concepts, revised the article and obtained funding. All authors read and approved the final manuscript.

\section{Ethics approval and consent to participate}

Not applicable.

\section{Patient consent for publication}

Not applicable.

\section{Competing interests}

The authors declare that they have no competing interests.

\section{References}

1. Shabalala S, Muller CJF, Louw J and Johnson R: Polyphenols, autophagy and doxorubicin-induced cardiotoxicity. Life Sci 180: 160-170, 2017. 
2. Damiani RM, Moura DJ, Viau CM, Caceres RA, Henriques JAP and Saffi J: Pathways of cardiac toxicity: Comparison between chemotherapeutic drugs doxorubicin and mitoxantrone. Arch Toxicol 90: 2063-2076, 2016.

3. Chatterjee K, Zhang J, Honbo N and Karliner JS: Doxorubicin cardiomyopathy. Cardiology 115: 155-162, 2010.

4. Li DL and Hill JA: Cardiomyocyte autophagy and cancer chemotherapy. J Mol Cell Cardiol 71: 54-61, 2014

5. Mitry MA and Edwards JG: Doxorubicin induced heart failure: Phenotype and molecular mechanisms. Int J Cardiol Heart Vasc 10: 17-24, 2016

6. Yang KC, Sathiyaseelan P, Ho C and Gorski SM: Evolution of tools and methods for monitoring autophagic flux in mammalian cells. Biochem Soc Trans 46: 97-110, 2018.

7. Xu X, Chen K, Kobayashi S, Timm D and Liang Q: Resveratrol attenuates doxorubicin-induced cardiomyocyte death via inhibition of p70 S6 kinase 1-mediated autophagy. J Pharmacol Exp Ther 341: 183-195, 2012.

8. Johnson R, Shabalala S, Louw J, Kappo AP and Muller CJF: Aspalathin Reverts Doxorubicin-Induced Cardiotoxicity through Increased Autophagy and Decreased Expression of p53/mTOR/p62 Signaling. Molecules 22: E1589, 2017.

9. Fuchs Y and Steller H: Programmed cell death in animal development and disease. Cell 147: 742-758, 2011.

10. Ryter SW, Mizumura K and Choi AM: The impact of autophagy on cell death modalities. Int J Cell Biol 2014: 502676, 2014.

11. Mizushima N, Levine B, Cuervo AM and Klionsky DJ: Autophagy fights disease through cellular self-digestion. Nature 451: 1069-1075, 2008

12. Nishida K, Yamaguchi O and Otsu K: Crosstalk between autophagy and apoptosis in heart disease. Circ Res 103: 343-351, 2008.

13. Eskelinen EL and Saftig P: Autophagy: A lysosomal degradation pathway with a central role in health and disease. Biochim Biophys Acta 1793: 664-673, 2009.

14. Liu B, Cheng Y, Liu Q, Bao JK and Yang JM: Autophagic pathways as new targets for cancer drug development. Acta Pharmacol Sin 31: 1154-1164, 2010.

15. Heras-Sandoval D, Pérez-Rojas JM, Hernández-Damián J and Pedraza-Chaverri J: The role of PI3K/AKT/mTOR pathway in the modulation of autophagy and the clearance of protein aggregates in neurodegeneration. Cell Signal 26: 2694-2701, 2014

16. Fasolo A and Sessa C: Targeting mTOR pathways in human malignancies. Curr Pharm Des 18: 2766-2777, 2012

17. Toyama EQ, Herzig S, Courchet J, Lewis TL Jr, Losón OC, Hellberg K, Young NP, Chen H, Polleux F, Chan DC, et al: Metabolism. AMP-activated protein kinase mediates mitochondrial fission in response to energy stress. Science 351: 275-281, 2016

18. Wong PM, Feng Y, Wang J, Shi R and Jiang X: Regulation of autophagy by coordinated action of mTORC1 and protein phosphatase 2A. Nat Commun 6: 8048, 2015.

19. Hung CM, Garcia-Haro L, Sparks CA and Guertin DA: mTOR-dependent cell survival mechanisms. Cold Spring Harb Perspect Biol 4: a008771, 2012.

20. Su M, Mei Y and Sinha S: Role of the Crosstalk between Autophagy and Apoptosis in Cancer. J Oncol 2013: 102735, 2013.

21. Cooper KF: Till Death Do Us Part: The Marriage of Autophagy and Apoptosis. Oxid Med Cell Longev 2018: 4701275, 2018.

22. Gump JM and Thorburn A: Autophagy and apoptosis: What is the connection? Trends Cell Biol 21: 387-392, 2011.

23. Chen S, Ren Q, Zhang J, Ye Y, Zhang Z, Xu Y, Guo M, $\mathrm{Ji} \mathrm{H}, \mathrm{Xu} \mathrm{C}, \mathrm{Gu} \mathrm{C}$, et al: N-acetyl-L-cysteine protects against cadmium-induced neuronal apoptosis by inhibiting ROS-dependent activation of Akt/mTOR pathway in mouse brain. Neuropathol Appl Neurobiol 40: 759-777, 2014.

24. Zhu J, Zheng C, Chen J, Luo J, Su B, Huang Y, Su W, Li Z and Cui T: Ghrelin protects human umbilical vein endothelial cells against high glucose-induced apoptosis via mTOR/P70S6K signaling pathway. Peptides 52: 23-28, 2014

25. Gu J, Hu W, Song ZP, Chen YG, Zhang DD and Wang CQ: Resveratrol-induced autophagy promotes survival and attenuates doxorubicin-induced cardiotoxicity. Int Immunopharmacol 32 : $1-7,2016$.

26. Reggiori F: 1. Membrane origin for autophagy. Curr Top Dev Biol 74: 1-30, 2006.

27. Xie Y, Kang R, Sun X, Zhong M, Huang J, Klionsky DJ and Tang D: Posttranslational modification of autophagy-related proteins in macroautophagy. Autophagy 11: 28-45, 2015.
28. Russell RC, Tian Y, Yuan H, Park HW, Chang YY, Kim J, Kim H, Neufeld TP, Dillin A and Guan KL: ULK1 induces autophagy by phosphorylating Beclin-1 and activating VPS34 lipid kinase. Nat Cell Biol 15: 741-750, 2013

29. Nakatogawa H: Two ubiquitin-like conjugation systems that mediate membrane formation during autophagy. Essays Biochem 55: 39-50, 2013.

30. Wang X, Wang XL, Chen HL, Wu D, Chen JX, Wang XX, Li RL, He JH, Mo L, Cen X, et al: Ghrelin inhibits doxorubicin cardiotoxicity by inhibiting excessive autophagy through AMPK and p38-MAPK. Biochem Pharmacol 88: 334-350, 2014.

31. Gu J, Fan YQ, Zhang HL, Pan JA, Yu JY,Zhang JF and Wang CQ Resveratrol suppresses doxorubicin-induced cardiotoxicity by disrupting E2F1 mediated autophagy inhibition and apoptosis promotion. Biochem Pharmacol 150: 202-213, 2018

32. Kobayashi S, Volden P, Timm D, Mao K, Xu X and Liang Q: Transcription factor GATA4 inhibits doxorubicin-induced autophagy and cardiomyocyte death. J Biol Chem 285: 793-804, 2010.

33. Bartlett JJ, Trivedi PC and Pulinilkunnil T: Autophagic dysregulation in doxorubicin cardiomyopathy. J Mol Cell Cardiol 104: 1-8, 2017.

34. Cappetta D, De Angelis A, Sapio L, Prezioso L, Illiano M, Quaini F, Rossi F, Berrino L, Naviglio S and Urbanek K: Oxidative Stress and Cellular Response to Doxorubicin: A Common Factor in the Complex Milieu of Anthracycline Cardiotoxicity. Oxid Med Cell Longev 2017: 1521020, 2017

35. Weerapana E, Wang C, Simon GM, Richter F, Khare S, Dillon MBD, Bachovchin DA, Mowen K, Baker D and Cravatt BF: Quantitative reactivity profiling predicts functional cysteines in proteomes. Nature 468: 790-795, 2010.

36. Li L, Chen Y and Gibson SB: Starvation-induced autophagy is regulated by mitochondrial reactive oxygen species leading to AMPK activation. Cell Signal 25: 50-65, 2013.

37. Bonet-Ponce L, Saez-Atienzar S, da Casa C, Sancho-Pelluz J, Barcia JM, Martinez-Gil N, Nava E, Jordan J, Romero FJ and Galindo MF: Rotenone Induces the Formation of 4-Hydroxynonenal Aggresomes. Role of ROS-Mediated Tubulin Hyperacetylation and Autophagic Flux Disruption. Mol Neurobiol 53: 6194-6208, 2016.

38. Kirkland RA, Adibhatla RM, Hatcher JF and Franklin JL: Loss of cardiolipin and mitochondria during programmed neuronal death: Evidence of a role for lipid peroxidation and autophagy. Neuroscience 115: 587-602, 2002.

39. Scherz-Shouval R, Shvets E, Fass E, Shorer H, Gil L and Elazar Z: Reactive oxygen species are essential for autophagy and specifically regulate the activity of Atg4. EMBO J 26: $1749-1760,2007$

40. Filomeni G, De Zio D and Cecconi F: Oxidative stress and autophagy: The clash between damage and metabolic needs. Cell Death Differ 22: 377-388, 2015.

41. Dodson M, Redmann M, Rajasekaran NS, Darley-Usmar V and Zhang J: KEAP1-NRF2 signalling and autophagy in protection against oxidative and reductive proteotoxicity. Biochem J 469: 347-355, 2015.

42. Mizunoe Y, Kobayashi M, Sudo Y, Watanabe S, Yasukawa H, Natori D, Hoshino A, Negishi A, Okita N, Komatsu M, et al: Trehalose protects against oxidative stress by regulating the Keap1-Nrf2 and autophagy pathways. Redox Biol 15: 115-124, 2018.

43. Zhang S, Liu X, Bawa-Khalfe T, Lu LS, Lyu YL, Liu LF and Yeh ET: Identification of the molecular basis of doxorubicin-induced cardiotoxicity. Nat Med 18: 1639-1642, 2012

44. Wallace KB: Doxorubicin-induced cardiac mitochondrionopathy. Pharmacol Toxicol 93: 105-115, 2003

45. Capranico G, Tinelli S, Austin CA, Fisher ML and Zunino F: Different patterns of gene expression of topoisomerase II isoforms in differentiated tissues during murine development. Biochim Biophys Acta 1132: 43-48, 1992.

46. Azuma Y, Arnaoutov A and Dasso M: SUMO-2/3 regulates topoisomerase II in mitosis. J Cell Biol 163: 477-487, 2003.

47. Wang M, Xu Y, Liu J, Ye J, Yuan W, Jiang H, Wang Z, Jiang $\mathrm{H}$ and Wan J: Recent Insights into the Biological Functions of Sestrins in Health and Disease. Cell Physiol Biochem 43: 1731-1741, 2017

48. Shaw RJ, Kosmatka M, Bardeesy N, Hurley RL, Witters LA, DePinho RA and Cantley LC: The tumor suppressor LKB1 kinase directly activates AMP-activated kinase and regulates apoptosis in response to energy stress. Proc Natl Acad Sci USA 101: 3329-3335, 2004 
49. Sancar A, Lindsey-Boltz LA, Unsal-Kaçmaz K and Linn S: Molecular mechanisms of mammalian DNA repair and the DNA damage checkpoints. Annu Rev Biochem 73: 39-85, 2004.

50. Sørensen CS, Hansen LT, Dziegielewski J, Syljuåsen RG, Lundin C, Bartek J and Helleday T: The cell-cycle checkpoint kinase Chk1 is required for mammalian homologous recombination repair. Nat Cell Biol 7: 195-201, 2005.

51. Liu EY, Xu N, O'Prey J, Lao LY, Joshi S, Long JS, O'Prey M, Croft DR, Beaumatin F, Baudot AD, et al: Loss of autophagy causes a synthetic lethal deficiency in DNA repair. Proc Natl Acad Sci USA 112: 773-778, 2015.

52. Abedin MJ, Wang D, McDonnell MA, Lehmann U and Kelekar A: Autophagy delays apoptotic death in breast cancer cells following DNA damage. Cell Death Differ 14: 500-510, 2007.

53. Monkkonen $\mathrm{T}$ and Debnath $\mathrm{J}$ : Inflammatory signaling cascades and autophagy in cancer. Autophagy 14: 190-198, 2018.

54. Qing G, Yan P and Xiao G: Hsp90 inhibition results in autophagy-mediated proteasome-independent degradation of IkappaB kinase (IKK). Cell Res 16: 895-901, 2006.

55. Salminen A, Hyttinen JMT, Kauppinen A and Kaarniranta K Context-Dependent Regulation of Autophagy by IKK-NF- $\kappa \mathrm{B}$ Signaling: Impact on the Aging Process. Int J Cell Biol 2012: 849541, 2012.
56. Niso-Santano M, Criollo A, Malik SA, Michaud M, Morselli E, Mariño G, Lachkar S, Galluzzi L, Maiuri MC and Kroemer G: Direct molecular interactions between Beclin 1 and the canonical $\mathrm{NF \kappa B}$ activation pathway. Autophagy 8: 268-270, 2012.

57. Lee HM, Shin DM, Yuk JM, Shi G, Choi DK, Lee SH, Huang SM, Kim JM, Kim CD, Lee JH, et al: Autophagy negatively regulates keratinocyte inflammatory responses via scaffolding protein p62/SQSTM1. J Immunol 186: 1248-1258, 2011.

58. Zhou HF, Yan H, Hu Y, Springer LE, Yang X, Wickline SA, Pan D, Lanza GM and Pham CT: Fumagillin prodrug nanotherapy suppresses macrophage inflammatory response via endothelial nitric oxide. ACS Nano 8: 7305-7317, 2014.

59. Fan M, Li Y, Yao C, Liu X, Liu J and Yu B: DC32, a Dihydroartemisinin Derivative, Ameliorates Collagen-Induced Arthritis Through an Nrf2-p62-Keap1 Feedback Loop. Front Immunol 9: 2762, 2018.

60. Bootman MD, Chehab T, Bultynck G, Parys JB and Rietdorf K: The regulation of autophagy by calcium signals: Do we have a consensus? Cell Calcium 70: 32-46, 2018.

(c) (i) (3) This work is licensed under a Creative Commons Attribution 4.0 International (CC BY-NC 4.0) License 